\title{
Wie kommen wir an die Bilder in unseren Köpfen? \\ Zur Methodologie einer interkulturell anwendbaren \\ Stereotypenerhebung
}

Stefan Ossenberg und Rupprecht S. Baur (Duisburg-Essen)

\begin{abstract}
SI.DE is an international research project at the University of Duisburg-Essen, which examines German, Russian, Turkish and Chinese auto- and heterostereotypes.

Questioning for the study is made online and consists of two surveys; one survey based on stereotypes and a second based on the perception of each other's foreign language. The questionnaire on stereotypes contains 140 attributes in total, while the respondents can select all of them without any limitation. The choice of these attributes is based on previous investigations, where methodologically formative surveys within the field of stereotype research had been compared with each other. Therefore, it is of high importance to take account of the transferability of the attributes to other languages, without any loss of crop-specific connotations. After the first phase of the study, the items on the attribute list have proved valuable. The construction of the questionnaire and the selected items are described in the following. Moreover, the perception in each other's language is being researched on the basis of polarity profiles.
\end{abstract}

\section{$1 \quad$ Einleitung}

Stereotype beeinflussen die Einstellungen gegenüber fremden Gruppen und Kulturen und gleichzeitig auch die Bereitschaft, die Sprache eines anderen Landes zu lernen. Es ist bekannt, dass es in der interkulturellen Kommunikation zu Störungen und Irritationen kommt, wenn Gesprächspartner unvorbereitet mit solchen negativen Stereotypen konfrontiert werden. Deshalb ist es wichtig, die Stereotype zu erforschen; denn nur auf der Grundlage dieser Erkenntnisse können Menschen verschiedener Länder und Kulturen auf interkulturelle Kontakte vorbereitet werden. Dabei geht es nicht nur um die Heterostereotype, sondern auch um Kenntnisse der Autostereotype, d. h. um das, was Völker über sich selbst denken.

Seitdem Walter Lippman 1922 sein Werk Public Opinion veröffentlicht hat, sind weltweit hunderte von Untersuchungen zu Stereotypen durchgeführt worden. Jedoch besteht bis heute noch immer keine im wissenschaftlichen Diskurs allgemein anerkannte Definition zum Begriff selbst. Während sich der Begriff Stereotyp ursprünglich aus dem Griechischen ableitet und als ,starres Muster ' übersetzt werden kann, ist er durch Lippman maßgeblich für die „Bilder in unseren Köpfen“ geprägt worden, auch wenn sich der Begriff Stereotyp seitdem je nach

Linguistik online 79, 5/2016 - http://dx.doi.org/10.13092/lo.79.3331

CC by 3.0 
wissenschaftlicher Ausrichtung und Forschungsrichtung in seiner Definition angepasst und weiterentwickelt hat.

Fest steht jedoch, dass alle aktuellen Definitionen zu Stereotypen auf dem Grundlagenwerk von Lippman basieren und sich an dessen Definition zumindest anlehnen. Einig sind sich somit alle aktuellen Definitionen zum Begriff des Stereotyps in einem Punkt: im Gegensatz zum Vorurteil, das meist emotionale Aspekte beinhaltet und oberflächliche Kenntnisse einer Kultur einseitig und extrem verallgemeinert, ist das Stereotyp ein kognitiver Wahrnehmungsprozess, der Unterscheidung und Verallgemeinerung $\mathrm{zu}$ anderen Gruppen zusammenfasst (cf. Florack 2007). Im bisherigen wissenschaftlichen und öffentlichen Diskurs gibt es jedoch keine deutlichen Trennlinien zwischen Klischee, Vorurteil und Stereotyp. ${ }^{1}$ Dabei kommt es gerade bei öffentlichen Diskursen oft zu Verwechslungen (cf. Löschmann/Strinska 1998: 733).

Neben der Erforschung geschlechtlicher, sozialer, beruflicher, religiöser und regionaler Stereotype besteht auch ein gesellschaftliches Interesse daran, nationale Stereotype zu erheben, um die Beziehungen zwischen Ländern, Nationen und Ethnien einschätzen und beeinflussen zu können. Jedoch steht die Stereotypenforschung auch hier vor einem Problem - dem einer einheitlichen und vergleichbaren Erhebungsmethode, die auch in transnationalen und interkulturellen Kontexten verwendet werden kann.

Seit der prägenden Studie von Katz und Braly (1933) wird das Merkmallisten-Verfahren (im Englischen als adjective checklist bezeichnet) ${ }^{2}$ als ein geeignetes Mittel für Erhebungen von Stereotypen angesehen, in Bezug auf die zur Auswahl zu stellenden Items herrscht dagegen noch immer Klärungsbedarf. Das Merkmallisten-Verfahren zur Erhebung von Stereotypen ist nicht unumstritten, wie bereits durch Manz (1968), Schäfer (1988), Eckes (2008), Thiele (2015) u. a. diskutiert. Jedoch werden abseits der Empfehlung für qualitative Erhebungsmethodiken durch alle Autoren, die sich mit der methodischen Problematik beschäftigen, keine substanziellen und praktisch anwendbaren Formen einer alternativen Erhebungsmöglichkeit bei ähnlich effektiver Operationalisierung und Forschungs-Tiefe aufgezeigt. Das gilt insbesondere in Bezug auf die Untersuchung, ,nationaler“ Stereotype (cf. Ganter 1997). Der seit Jahrzehnten geführte wissenschaftliche Disput zu Methoden in der Stereotypenforschung vernachlässigt elementare Fragen, wenn er die epistemologischen Komponenten in den Vordergrund stellt. Die Kritik an den quantiativ orientierten Untersuchungen - je nachdem welcher Forschungsmeinung gefolgt wird, divergiert diese Kritik - wird häufig an den grundlegenden ersten Erhebungen fest gemacht, die den heutigen methodologischen Standards nur noch teilweise entsprechen. Mängel der Konzeption und daraus resultierende angreifbare Ergebnisse werden dann der Methode an sich zugeschrieben. Die forschungsmethodologische Innovation der jeweiligen Studien wird jedoch durch diese Metaanalysen, die Forschungsentwicklungen auf epistemologischet Ebene analyiseren, verstellt.

\footnotetext{
${ }^{1}$ Eine gute Einordnung und Verortung bietet Hahn mit seinen „12 Thesen zur Stereotypenforschung“ (Hahn/Mannová 2007: 15-24).

${ }^{2}$ Aufgrund der sehr divergenten Forschungslinien und ihrer Interessen und der dabei jeweils eigenständigen Weiterentwicklung der amerikanischen und deutschen Stereotype-Forschung plädieren wir für eine Unterscheidung von ,adjective cheklist“ und „Merkmallisten-Verfahren“, damit eine linguistisch erkennbare Differenzierung zwischen den Forschungsentwicklungen möglich ist.
} 
Um die Erhebung und Messung von Stereotypen nach dem Merkmallistenverfahren operativ zu verbessern, wäre es aus sowohl aufgrund der o. a. Gründe als auch aus den im Folgenden genannten Gründen wünschenswert, ein transnational und interkulturell anwendbares Erhebungsinstrument zu erstellen und damit die Erhebungsmöglichkeiten auf dem Gebiet der Stereotypenorschung weiter zu entwickeln.

Bisherige Befragungen mit dem Merkmallisten-Verfahren arbeiten mit unterschiedlichen Fragen und Antwortoptionen. Die Ergebnisse der einzelnen Studien sind dadurch nur sehr eingeschränkt miteinander vergleichbar. Die erste deutsche Liste von Sodhi und Bergius (1953) enthält z. B. 207 Eigenschaften, die neueren Listen von Apeltauer (2002) 178 und von Grünewald (2005) 147, die Liste von Baur und Ossenberg (2014) 140 Eigenschaften. $^{3}$

Aus Gründen der Vergleichbarkeit sollte eine Merkmalliste zu einem hohen Prozentsatz für alle Sprachen inhaltlich gleich sein. Die Übersetzbarkeit bzw. Äquivalenz der Merkmale in verschiedenen Sprachen muss überprüft und abgesichert werden, um kulturvergleichende Auswertungen zu ermöglichen. So gibt es Begriffe, für die es keine passende Äquivalenz zu geben scheint, so zum Beispiel bei der Eigenschaft ,philosophische Lebenshaltung' für das Chinesische, oder für ,konventionell' im Russischen.

Kulturspezifische Konnotationen einzelner Begriffe müssen aus diesem Grund berücksichtigt und ein ausgewogenes Verhältnis zwischen ,neutral', ,positiv‘ oder ,negativ' wahrgenommenen Eigenschaften geschaffen werden. (cf. hierzu Abschnitt 3.1) So konnten wir beispielsweise feststellen, dass der Begriff sparsam als Eigenschaft der Deutschen bei Russen und Türken eher negativ in Richtung, geizig' konnotiert ist. Umgekehrt ist die Eigenschaft nationalstolz in Deutschen eher negativ konnotiert, im Chinesischen, Russischen und Türkischen dagegen positiv.

Nach bisherigen Untersuchungen zu Auto- und Heterostereotypen, also den „Selbst“- und „Fremdbildern“ im Forschungsprojekt SI.DE an der Universität Duisburg-Essen hat sich die hier vorgestellte 140 Eigenschaften umfassende Liste zur Erhebung nationaler Stereotype bewährt. Bei ihrer Entwicklung wurde versucht, die o. a. Kriterien zu berücksichtigen. Sie ist sowohl im deutschen, chinesischen russischen und türkischen Sprachraum angewendet und auf ihre kulturkontrastive und interkulturelle Funktionalität in allen vier Sprachen überprüft worden. Sie wurde auf Grundlage der Merkmallisten und Untersuchungen von Katz/Braly (1933), Sodhi und Bergius (1953), Apeltauer (2002) und Grünewald (2005) entwickelt, die in der Tradition des Merkmallisten-Verfahrens stehen, das sich in der Forschung des Fachs „Deutsch als Fremdsprache“ und „Deutsch Zweitsprache“ entwickelt hat. Wir sind der Meinung, dass es zukünftig sinnvoll ist, Stereotype im Rahmen wechselseitiger Befragungen, also unter Einbeziehung beider „nationaler“ Eigen- und Fremdperspektiven, durchzuführen, weil dadurch interkulturelle Fragestellungen ,vielseitiger“ analysiert, interpretiert und bewertet werden können.

\footnotetext{
${ }^{3}$ Die Eigenschaftenliste von Baur und Ossenberg (2014) findet sich sowohl im Anhang, als auch auf der Internetseite des Stereotypen-Projekts: https://side.uni-due.de [17.03.2016].
} 


\section{$2 \quad$ Zum Stand und Vergleich bisheriger Forschungen}

Während sich Walter Lippmann, der den Begriff des Stereotyps maßgeblich geprägt hat, mit der Frage beschäftigt, aus welchem Grund Stereotype existieren und welchen Nutzen sie für den Menschen haben, und dies an den drei wesentlichen Aspekten „ökonomische Ursachen“, „Verteidigungsmittel“ und „Selbstschutz" festmacht, beschäftigen sich die forschungsorientierten Studien, die auf Lippmans Annahmen aufbauen, mit der Frage, welche Stereotype generell existieren. Stilbildend ist dabei die Befragung der beiden amerikanischen Soziologen Daniel Katz und Kenneth Braly (1933), auf die sich direkt oder indirekt alle Folgestudien zu nationalen Stereotypen beziehen.

„Racial stereotypes of one hundred College students“ aus dem Jahr 1933 geht der Frage nach, ob Stereotype, die von einer gesellschaftlichen Gruppe anderen sozialen oder ethnischen Gruppen zugeschrieben werden, auf konkreten Erfahrungen mit einzelnen Mitgliedern dieser Gruppen basieren oder ob die zugeschriebenen Eigenschaften als Stereotype bzw. Vorurteile durch einen Gruppenkonsens entstehen. Die Methode, die Katz und Braly dabei etablierten, wird auch als „Eigenschaftslisten-Verfahren“ bezeichnet. Um die Selektionsmerkmale für das Experiment zu bestimmen, wurden in einem Pretest 25 Studenten dazu aufgefordert, spezifische Merkmale oder Eigenschaften zu nennen, die nach Meinung der Probanden für die ethnische Gruppen der Deutschen, Italiener, Neger [sic!], Iren, Engländer, Juden [sic!], Amerikaner, Chinesen, Japaner und Türken typisch sind. Diese Nennungen wurden zusammengeführt und bildeten die Grundlage für diese erste zu Forschungszwecken erstellte Merkmalliste, die 84 Adjektive umfasste. Während sich die amerikanische Stereotypenforschung aufbauend auf dieser Studie vor allem mit nationalen Stereotypen im eigenen Land (also den Bildern in den Köpfen zu Migrationsgruppen) beschäftigt (cf. u. a. Gilbert 1951; Coffman/Walter 1969), fokussiert die deutschsprachige Stereotypenforschung vor allem nationale Stereotype im Bezug auf andere Länder.

Im Jahr 1953 greifen die beiden Sozialpsychologen Kripal Singh Sodhi und Rolf Bergius die Studie von Katz und Braly auf und adaptieren diese für eine Befragung im deutschen Kontext. Sodhi und Bergius geht es dabei um die Frage

[...] welchen Kreis von Vorstellungen deutsche Menschen einer bestimmten sozialen Schicht von 14 europäischen und außereuropäischen Völkern haben von den Amerikanern, amerikanische Negern, Chinesen, Deutschen, Engländern, Franzosen, Indern, Italienern, Juden, Polen, Russen, Spaniern, Tschechen und Türken.

(Sodhi/Bergius 1953: 15)

Die Wahl der Völkergruppen wurde nach Angaben von Sodhi und Bergius bewusst nach zwei Kategorien ausgearbeitet. Um die durch die jüngere Geschichte und Politik geprägten ethnischen Vorstellungen der Deutschen zu diesem Zeitpunkt zu erkennen zu können, wurden zum einen nationale Gruppen ausgesucht, mit denen in der Zeit des Dritten Reichs und nach Kriegsende intensive Kontakte bestanden, zum anderen nationale Gruppen ohne einen solchen Kontakt (Inder, Chinesen). Dabei folgen die beiden Sozialpsychologen der StereotypeDefinition von Kimball Young und definieren Stereotyp als 
ein falsch klassifizierender Begriff, mit dem im Allgemeinen irgendein starker Gefühlston des Mögens oder Nichtmögens, der Anerkennung oder Ablehnung verbunden ist. Die geringe Übereinstimmung der Stereotypen mit der Wirklichkeit ist also Folge einer Verallgemeinerung von ein oder zwei Einzelfällen.

(Sodhi/Bergius 1953: 19)

Während sich Katz und Braly auf eine Eigenschaftenliste mit 84 Begriffen beschränkten und nur 100 Amerikaner (ohne Erhebung soziodemographischer Faktoren) befragten, erweiterten Sodhi und Bergius die Eigenschaftsliste auf 207 Items, um die Problematik der Polysemie der ,weitgehenden Modifizierbarkeit der Wortbedeutung durch die Beschaffenheit des Zusammenhanges“ (Sodhi/Bergius 1953: 25) - einzugrenzen. Befragt wurden 442 männliche und 439 weibliche Deutsche mit Hilfe von Studierenden. Dabei sollte jeder Studierende zwei von den insgesamt 14 verschiedenen ethnischen (damals als „völkisch“ bezeichneten) Gruppen erheben, um dadurch die Untersuchung qualitativ kontrollieren zu können und zuverlässigere Ergebnisse zu erzielen. Obwohl in der Studie insgesamt 881 Personen befragt wurden, reduzierte sich die Anzahl der Befragten für die einzelnen ethnischen Gruppen dadurch erheblich, so dass die Probandenzahl von Katz und Braly nur in wenigen Einzelfällen übertroffen wurde.

Diese Modifikation gegenüber der amerikanischen Versuchsanordnung war notwendig, weil eine Bearbeitung von 14 Eigenschaftslisten für die Versuchsperson viel zu ermüdend gewesen wäre [...]. Wenn ein Mensch für alle 14 völkische Gruppen spezifische Charakteristika hätte angeben sollen, wäre er sicherlich davor zurückgeschreckt, sich hinsichtlich der Bezeichnungen zu oft zu wiederholen.

(Sodhi/Bergius 1953: 33)

Diese zu Recht befürchtete „Ermüdung“ ist der großen Anzahl von 207 Eigenschaften geschuldet. Der Grund dafür liegt darin, dass Sodhi und Bergius eine Vergleichbarkeit mit der Studie von Katz und Braly erhalten wollten, aber bei der Übertragung der amerikanischen Liste ins Deutsche auf eine Fülle von Übersetzungsproblemen stießen, die zu einer größeren Anzahl an Items führten. So gibt es beispielsweise für das Wort shrewd eine ganze Anzahl verschiedener Entsprechungen, die sowohl als positiv ,schlau' oder aber negativ als ,hinterlistig' ins Deutsche übertragen werden können. Entsprechungen, die in Wörterbüchern aufgeführt werden, sind im Sinn von ,schlau' gewitzt, scharfsinnig, gewandt, pfiffig, im Sinn von ,hinterlistig' gerissen, durchtrieben, arglistig, raffiniert; als ,neutral' können die Übersetzungsäquivalente bauernschlau und geschäftstüchtig angesehen werden. Ohne eine Dokumentation der Konnotationen fällt es schwer, eine in mehrere Sprachen zu übersetzende Merkmalliste zu erstellen. Wie bereits oben erwähnt wurde, entschieden sich Sodhi und Bergius deshalb dazu, mehrere Übersetzungsoptionen eines englischsprachigen Begriffes in ihre Liste aufzunehmen.

Dabei ließen sie jede Eigenschaft bewerten und errechneten aus den Einzelwerten für jede Bezeichnung den Durchschnittswert. Dabei stellten sie eine Werteskala von 1-11 zur Verfügung, wobei die Zahl 1 die schlechteste und die Zahl 11 die beste Bewertung darstellte. Mithilfe mathematischer Berechnungen konnten die Psychologen Gefühlswerte zusammenfassen, um so ein Gesamturteil für jedes der 14 Völker hinsichtlich des gefühlsmäßigen $\mathrm{Zu}$ - oder Abneigungsgrades treffen zu können. 
Die Merkmalliste von Sodhi und Bergius ist für den deutschsprachigen Raum genauso prägend, wie die Studie von Katz und Braly es für den amerikanischen Kulturraum ist. Auch Ernst Apeltauer (2002) greift diese Liste auf, indem er sich darüber hinaus auf die Merkmalliste von Keller (1986) bezieht, der sich ebenfalls von Sodhi und Bergius inspirieren ließ. Bei der Erkundung der Einstellung von norwegischen Schülern zu Deutschen verwendet Apeltauer zusätzlich zu seiner Merkmalliste qualitative Methoden, um durch eine Triangulation der Daten zu besser abgesicherten Aussagen zu kommen. Allerdings relativiert sich sein Anspruch durch die geringe Anzahl seiner Probanden (s. u.). Apeltauer geht davon aus, dass besonders die Fremd- und Selbstbilder einer Gruppe die Verständigung zwischen verschiedenen ethnischen Gruppen beeinflussen. Um dieser Frage nachzugehen, befragt der Fremdsprachenforscher insgesamt 163 Probanden an einer norwegischen Schule, davon 156 Gymnasialschüler und sieben Lehrende.

Apeltauer geht von drei Hypothesen aus:

1. Gymnasialschüler stützen sich auf prototypische Formen von Stereotypen.

2. Geringe Eigenerfahrung und kaum Kontakt mit Mitgliedern fremder Gruppen führen zu Beurteilungen, die auf kollektivem Wissen beruhen.

3. Durch Erfahrungen und Erlebnisse differenziert sich das prototypische Bild mit zunehmendem Alter und führt zu individuellen Veränderungen bei der Einschätzung von Stereotypen.

Apeltauers Studie mit 178 Eigenschaften ist methodisch anregend, sie kann jedoch aufgrund der eingeschränkten Anzahl Befragter keine repräsentativen Aussagen liefern.

Die jüngste Studie, die als forschungsprägend angesehen werden kann, wurde 2005 von Matthias Grünewald veröffentlicht und beschäftigt sich mit dem Deutschlandbild von japanischen Deutschlernern. In seiner Untersuchung wird keine ungeprüfte Übernahme der in früheren Untersuchungen verwendeten Eigenschaften vorgenommen, sondern Grünewald führt, wie Katz und Braly, zur Gewinnung der Eigenschaften eine eigenständige Voruntersuchung durch. Erstmalig kommt dadurch beispielweise die Eigenschaft umweltbewusst in die Stereotypenforschung.

Es ist allerdings festzustellen, dass der überwiegende Teil der Eigenschaften, die Grünewald in seiner Erhebung nutzt, im Endeffekt auch schon bei Apeltauer zu finden ist. Seine Arbeit ist jedoch mit Blick auf die kontrastive Landeskunde in Bereich von Deutsch als Fremdsprache für den asiatischen Raum als außerordentlich nützlich einzuordnen. Durch eine Kombination von geschlossenen, offenen und halboffenen Fragen gelingt es Grünewald, Daten zu gewinnen, die eine Grundlage für detaillierte Analysen und Interpretationen zum Deutschlandbild in Japan bieten. Die Antworten der 659 Befragten plus einer Kontrollgruppe von 59 Studierenden, die alle an der Universität Matsuyama in Südjapan studierten, zeigen ein sehr positives Deutschlandbild dieser Untersuchungsgruppe. Dies ist allerdings unter anderem durch die besondere Interessenslage der Befragten zu begründen, die zum Zeitpunkt von Grünewalds Erhebung alle ein Studium des Deutschen als zweite Fremdsprache gewählt hatten.

Auch bei Grünewald rückt, ähnlich wie bei Sodhi und Bergius, die Übersetzungsproblematik gewisser Items in den Fokus des methodischen Aufbaus der Merkmalliste. Grünewald nutzt 
hier bei schwierig zu übersetzenden Merkmalen den Diskurs mit Muttersprachlern und erarbeitet mit diesen gemeinsam zu 20 problematischen Begriffen jeweils drei Definitionen, die sowohl Konnotationen enthalten, als auch Umschreibungen beinhalten. Dieses Vorgehen ist aus unserer Sicht zielführend, allerdings halten wir es für notwendig, in dieser Weise nicht nur für ausgewählte Items, sondern bei allen Items einer Merkmalliste vorzugehen.

Natürlich existieren zahlreiche andere Studien zu ,nationalen“ Stereotypen. Jedoch sind die oben genannten Studien stilprägend. An Ihnen orientieren sich die meisten der existierenden Erhebungen, die ein Merkmallisten-Verfahren verwenden. Aus diesem Grund werden hier nur diese vier Studien angeführt. Eine ausführliche Analyse aller relevanten Studien im deutschsprachigen Raum wird in weiteren Publikationen erfolgen.

Ein direkter Vergleich der bereits zuvor genannten vier Eigenschaftenlisten, ihrer Entstehungszeit, der Anzahl der Items, die Anzahl der Befragten und die Zahl der abgefragten Ethnien, zu denen gefragt wird, ist der Tabelle $1 \mathrm{zu}$ entnehmen.

\begin{tabular}{|l|l|l|l|l|}
\hline & Katz \& Braly & Sodhi \& Bergius & Apeltauer & Grünewald \\
\hline Jahr & 1933 & 1953 & 2002 & 2005 \\
\hline Eigenschaften & 84 & 207 & 178 & 147 \\
\hline Befragte & 100 & 881 & 163 & 659 \\
\hline abgefragte Ethnien & 10 & 14 & 1 & 1 \\
\hline
\end{tabular}

Tabelle 1: Vergleich bisheriger Studien

Während die Studie von Katz und Braly durch die Einführung des EigenschaftenlistenVerfahrens für die weitere Forschungen bedeutend ist, zeichnet sich die Berliner Studie von Sodhi und Bergius durch die Sensibilisierung für die Polysemie von Begriffen und die damit verbundene Diskussion der Übersetzungsproblematik aus, während Apeltauer die Triangulation zur qualitativen Interpretation genannter Eigenschaften in die nationale Stereotypenforschung einbringt. Grünewalds Studie unterscheidet sich von Apeltauer durch ihre Eigenständigkeit im japanischen Kontext und zeigt exemplarisch, dass in nicht westlich orientierten Staaten andere Stereotype zu Deutschen und Deutschland existieren, als im europäischen und nordamerikanischen Raum.

Bei den drei Studien von Katz und Braly, Sodhi und Bergius sowie Apeltauer ist festzustellen, dass zum Teil historisch geprägte Begriffe unhinterfragt übernommen werden. Die an die Zeit gebundene Relativität gewisser Begrifflichkeiten wird in allen hier besprochenen Studien nicht thematisiert. So stammen die noch heute in den Studien verwendeten Begriffe abergläubisch, naiv, kindlich und faul aus der amerikanischen Studie von 1933, wo sie den „Negroes“ zugeschrieben wurden; die Eigenschaft ,brutal' stammt aus der Studie von Katz und Braly und wurde dort - vermutlich in Zusammenhang mit den Kämpfen um die Dardanellen im Ersten Weltkrieg - den Türken zugeschrieben; ,impulsiv` stammt aus derselben Studien von 1933 und bezog sich auf die Italiener. Generell lässt sich feststellen, dass die zeitlich und kulturell bedingten Wertungen, die sich in den Eigenschaften widerspiegeln, bei der Übernahme in die Merkmallisten i. d. R. nicht berücksichtigt werden.

Bei den neueren Studien zum Deutschlandbild von Apeltauer und Grünewald gibt es keine 
Wechselseitigkeit der Befragungen. Wir betonen noch einmal, dass wir es zur Vorbereitung auf interkulturelle Kontakte für wichtig halten, Heterostereotypen der Deutschen und die Autostereotypen der anderen involvierten ethnischen Gruppe zu kennen und diese Kenntnis auch wechselseitig zu vermitteln.

Bei der Erstellung von Merkmallisten sollten zudem nicht nur Eigenschaften aus der Stereotypenforschung, sondern beispielsweise auch Items aus aktuellen Studien zur Meinungsforschung verschiedener Institutionen berücksichtigt werden (cf. dazu Abschnitt 3.1).

Wichtig erscheint es uns auch, die Perspektive einer longitudinalen Konzeption zu eröffnen. Aktuelle Ereignisse - wie Konflikte, die zu kriegerischen Auseinandersetzungen führen können die Einstellungen zwischen Völkern, Staaten oder ethnischen Gruppen sehr plötzlich verändern. Solche Veränderungen lassen sich durch eine interkulturell erprobte Eigenschaftsliste im Rahmen des von uns erprobten online-Verfahrens zeitnah und zuverlässig überprüfen. ${ }^{4}$

\section{$3 \quad$ Erstellung einer, ,interkulturellen“ Eigenschaftenliste}

\subsection{Eigenschaftenauswahl}

Aus den oben angeführten Gründen erscheint uns ein Abgleich mit den bisher genutzten stilprägenden Merkmallisten und ihren Eigenschaften notwendig. Offene Befragungen, um eigene Merkmallisten zu erstellen (wie beispielsweise die Eigenschaftenliste von Grünewald), haben den Nachteil, dass sie sehr aufwendig sind, aber den Vorteil, dass ggf. neue Eigenschaften genannt werden. Bei Grünewald war das v. a. die Eigenschaft umweltbewusst, wie wir bereits erwähnt haben. Prinzipiell ist es wünschenswert, Befragungen mit größeren Probandengruppen durchzuführen, um zuverlässige Werte zu erhalten, aber auch hier kann es zu Verzerrungen kommen, wenn nur wenige Items zur Wahl stehen. ${ }^{5}$

Das Sammeln spontaner Assoziationen von Probanden zu einem Land und einer Ethnie ist eine gern genutzte Methode. Bei solchen Erhebungen beziehen sich sehr viele Assoziationen auf das Land selbst (z. B. landschaftliche und geografische Merkmale); deshalb ist die ,Ausbeute' für Eigenschaften, welche die Einstellung zu den Menschen ausdrücken, i. d. R. gering (wie sich auch in der FORSA-Studie 2007 gezeigt hat). Die Aufnahme solcher „Merkmale“ in Eigenschaftslisten, mit denen Einstellungen zu anderen Sprachen und Kulturräumen erhoben werden sollen, ist nicht möglich.

Aus dem Gesagten ist ersichtlich, dass die Untersuchungsmethoden und Ergebnisse der einzelnen Studien genau untersucht und kritisch bewertet werden müssen, bevor neu gefundene Eigenschaften in eine interkulturell anwendbare Merkmalliste aufgenommen werden können. Es hat sich deshalb als zielführend erwiesen, als Ausgangspunkt für die Erstellung einer Merkmalliste bisherige Stereotypenstudien und die dort verwendeten Eigenschaften, die in unterschiedlichen Kulturräumen entstanden sind, miteinander zu vergleichen.

\footnotetext{
${ }^{4}$ Gegenwärtig überprüfen wir in diesem Zusammenhang, ob sich durch die Ukraine-Krise die Einstellung der Deutschen gegenüber den Russen und/oder Russland verändert hat.

${ }^{5}$ Dieses Phänomen zeigt sich bei manchen Studien zu Stereotypen, so wie exemplarisch bei der FORSA-Studie zum Russlandbild der Deutschen, wie von uns bereits gezeigt wurde (Baur et al. 2013).
} 
In unserer inhaltlichen Metaanalyse haben wir diesen Vergleich vorgenommen. Dabei stellte sich heraus, dass sich die in den verschiedenen Merkmallisten enthaltenen Eigenschaften im Kern nur wenig voreinander unterscheiden. ${ }^{6}$ Bei unserer Analyse haben wir uns deshalb dazu entschieden, im ersten Schritt der Erstellung einer interkulturell anwendbaren Erhebung die Begrifflichkeiten der einzelnen Listen zu clustern und dabei Entscheidungen getroffen, die wir im Folgenden exemplarisch darstellen. Diese Auswahl ist maßgeblich durch folgende drei Kriterien bestimmt worden:

1. Das Vorkommen des entsprechenden Items in den prägenden Studien.

2. Die Übertragbarkeit der einzelnen Eigenschaften in die Sprachen Englisch, Russisch, Türkisch und Chinesisch.

3. Die Überprüfung der Konnotationen der ausgewählten Begriffe in den involvierten Sprachen im Vergleich zum Deutschen.

Auch wenn eine zeitliche Relativität gewisser Begrifflichkeiten nicht von der Hand zu weisen ist, so gibt es eine große Menge an Eigenschaften, die sich in den vergangenen Jahrzehnten bei Befragungen bewährt hat. Allerdings finden sich vor allem bei Sodhi und Bergius (1953) noch viele Begriffe, die aus verschiedenen Gründen heute nicht mehr passen und die in unseren Voruntersuchungen von Probanden auch nicht mehr genannt wurden. Hier wären folgende Beispiele zu nennen: die „Eigenschaft“ vom rostigen Nagel zur Million (damals als Übersetzung für das Steretoyp vom Tellerwäscher zum Millionär gewählt), starker Körpergeruch oder krumme Nase (Eigenschaften die von Sodhi und Bergius aus der Merkmalliste von Katz und Braly (1933) zu „Negroes“ bzw. „Jews“ übernommen wurden)7. Die Begriffe Antikommunisten, antisemitisch und der beste Soldat übernimmt Apeltauer (2002) von Keller (1986) bzw. Sodhi und Bergius. Während es sich bei Antikommunisten um einen aus dem Kalten Krieg stammenden Begriff handelt, den Apeltauer ohne Diskussion von Keller übernimmt, und der mittlerweile aufgrund der politischen Weltlage als überholt angesehen werden kann, ist antisemitisch ein Terminus, der in der asiatischen Welt nicht verstanden wird. Obwohl die Unterschiede im westlichen Diskurs klar sind, haben wir uns für fremdenfeindlich und rassistisch als in gewisser Weise übergeordnete Begriffe entschieden. Auf welche ethnischen Gruppen sich diese Qualifikative in den verschiedenen Befragungen beziehen, kann durch qualitative Befragungen erhoben werden. Die Begrifflichkeit der beste Soldat ist sprachlich antiquiert, und wurde von uns durch das Item gute Soldaten ausgetauscht.

Auch der Begriff hübsche Frauen den Apeltauer ebenfalls aus der Eigenschaftenliste der beiden Berliner Sozialpsychologen übernommen hat, sollte ergänzt werden, denn es fehlt in bisherigen Befragungen ein männliches Pendant. Wir haben uns dafür entschieden nicht den Terminus hübsche Männer zu wählen, da hier kulturspezifisch bedingte Missverständnisse

\footnotetext{
${ }^{6}$ In der Studie von Grünewald (2005) erscheinen einige weitere Eigenschaften. Dies scheint aber dadurch bedingt zu sein, dass die Befragten durch den Deutschunterricht und das verwendete Lehrwerk neue Bilder aufbauten, die von Grünewald untersucht wurden. Ein Schwerpunkt der Studie liegt damit auf der Erforschung der Einflüsse von Lehrwerken von Deutsch als Fremdsprache auf stereotype Vorstellungen japanischer Studierender. Grünewald (2005: 194) selbst räumt ein, dass seine Untersuchung „Begrenzungen“ unterlag, die „nur teilweise die Erfüllung der Gütekriterien“ erreichte.

${ }^{7}$ Dieser und ähnliche Begriffe sollten und können immer nur im Kontext der damaligen Zeit interpretiert und analysiert werden. Jedoch zeigt sich hier exemplarisch die Problematik der historischen Tradierung bei gewählten Merkmalen.
} 
aufkommen könnten, sondern das Item attraktive Männer eingeführt, da hier zweideutige Konnotationen bei der Übertragung in andere Sprache weitgehend ausgeschlossen zu sein scheinen.

Nachdem wir die einzelnen Studien und ihre Eigenschaften miteinander verglichen und dabei bereits eine große Zahl an Eigenschaften aufgrund der beispielhaft erläuterten Faktoren ausgeschlossen hatten, überprüften wir in einem weiteren Schritt aktuelle Studien von Meinungsforschungsinstituten, um eventuell weitere einzuarbeitende Eigenschaften aufzufinden.

Dazu gehörten u. a. die FORSA-Studie (Forsa 2007) ${ }^{8}$, die Deutschland-Liste (Goethe-Institut 2011), die OeTTINGER Deutschland-Studie (Rheingold Salon 2012) und die BertelsmannStudie Im Osten was Neues? (Kucharczyk et al. 2013). Wir wählten dabei Studien aus, die methodisch unterschiedlich vorgingen und die in der Presse eingehend diskutiert wurden. So wurde bei der FORSA-Studie auf Telefoninterviews zurückgegriffen, die Deutschlandliste nutzte Online-Fragebögen mit ausschließlich offenen Antwortmöglichkeiten, die OeTTINGER-Studie nutzte eine Mischung aus Einzel- und Gruppeninterviews und die BertelsmannStudie setzte auf Likert-Skalen zur Messung der Stärke vorgegebener Eigenschaften. Als Ergänzungen zu den bereits besprochenen wissenschaftlichen Studien wurden aus der FORSAStudie, die sich auf das deutsch-russische Meinungsbild bezieht, die Eigenschaften ,trinkfreudig' und ,friedliebend' hinzugefügt.

Im Gegensatz zu der in der FORSA-Studie vorkommenden negativ konnotierten Eigenschaft ,trinkfest', die als Euphemismus für Alkoholismus angesehen werden kann, haben wir in unsere Liste ,trinkfreudig' aufgenommen. Diese Eigenschaft wird im deutschen Sprachgebrauch eher als neutral bis positiv bewertet - es schwingen Assoziationen von geselligem Verhalten mit.

Auf eine tiefergehende Analyse der einzelnen Studien soll an dieser Stelle verzichtet werden, jedoch nicht unerwähnt bleiben, dass alle Studien sowohl im Bezug auf ihre jeweilige Methodik, als auch auf die abgefragten Inhalte Schwächen haben und Mängel aufweisen, was sich bei von uns durchgeführten Reproduktions-Simulationen gezeigt hat. Diese haben wir in den vergangenen drei Jahren mit insgesamt über 200 Teilnehmern durchgeführt.

Nach Aufarbeitung der relevanten Studien und Erhebungen der Meinungsforschungsinstitute gingen wir einen dritten Schritt - den der Übersetzung in andere Sprachen, um ausschließen zu können, dass sich in unserer Merkmalliste Begriffe befinden, die nur schwer anderen Sprachen und Kulturen verstanden werden können. Hier stellt sich das Problem, ein Äquivalent in der anderen Sprache zu finden, das dem deutschen Begriff auch konnotativ nahe kommt. Nach einem Vergleich mit dem Englischen, der sich als wenig problematisch erwies, überprüften wir das Russische ${ }^{9}$, das Türkische ${ }^{10}$ und das Chinesische ${ }^{11}$.

\footnotetext{
${ }^{8}$ Eine Analyse und ausführliche Diskussion der dort genutzten Merkmale wurde von uns bereits 2013 durchgeführt (cf. Baur et al. 2013).

${ }^{9}$ Hier gilt unser Dank Marina Zarudko für ihre Unterstützung bei Übersetzungen, der Durchführung qualitativer Gruppeninterviews und Klärung von Übersetzungsunstimmigkeiten.

${ }^{10}$ Beteiligt am Prozess der Übersetzung und Konnotationsermittlung ins Türkische waren Haci-Halil Usluçan, Leyla Coşan, Yasemin Balcı, Hasan Bolat, Mustafa Çakır, Sevinç Sakarya Maden, Ahmet Uğur Nalcıoğlu, Mustafa Özdemir, Ali Osman Öztürk und Cemal Yıldız.
} 
Da Einwanderer aus der Türkei und Russland die beiden größten Migrationsgruppen in Deutschland darstellen, scheint es nach unserer Auffassung außerordentlich interessant, sowohl Erhebungen in diesen beiden Ländern als auch Erhebungen bei den respektiven Zuwanderungsgruppen selbst durchzuführen. Als weitere Sprache wählten wir das Chinesische, um an die Erfahrung von Grünewald anschließend einen ,asiatische Blickwinkel“ zu berücksichtigen und dadurch die interkulturelle Gültigkeit unserer Eigenschaftenliste breiter abzusichern.

Die Äquivalenzproblematik lässt sich am Beispiel der Eigenschaft ,sparsam‘ illustrieren. Während es im Deutschen eine Differenzierung zwischen den Termini sparsam und geizig gibt, wobei die eine Eigenschaft positiv, die andere dagegen negativ konnotiert wird, ist diese Differenzierung sowohl im Russischen, als auch im Türkischen sowie auch im Chinesischen nicht so eindeutig. In allen drei Sprachen kann ,sparsam‘ vielmehr als Synonym für geizig, also negativ konnotiert sein. Während die autostereotype Zuschreibung der Deutschen als ,sparsam‘ eindeutig positiv ist, sind bei Befragungen in anderen Ländern die Nennungen ,sparsam‘ und ,geizig' gemeinsam in einem spezifischen Zusammenhang zu interpretieren und zu analysieren. Um solche und ähnliche Konnotationsübertragungen festzustellen, haben wir alle Items unserer Eigenschaftenliste mit bilingualen Sprachwissenschaftlern aus den jeweiligen Kultur- und Sprachräumen in Gruppengesprächen diskutiert und die Ergebnisse als Grundlage für spätere Interpretationen und vertiefende qualitative Untersuchungen festgehalten.

Wie wir exemplarisch am obigen Beispiel gezeigt haben, können die Konnotationen für Eigenschaften in den involvierten Sprachen und Kulturen divergieren. Die Konnotationen für alle Eigenschaften wurden deshalb in unserem Projekt jeweils im Deutschen, Russischen, Türkischen und Chinesischen mit der Bewertung ,positiv', ,negativ` oder ,neutral ‘ versehen, um feststellen zu können, wo Divergenzen bestehen, die bei späteren Auswertungen und Interpretationen berücksichtigt werden müssen.

Neben einer Erhebung der Eigenschaften als Auto- und Heterostereotype in den genannten Ethnien, möchten wir auch herausfinden, ob und ggf. bei welchen soziodemographischen Faktoren sich Unterschiede in den Stereotypen zeigen.

\section{$4 \quad$ Ausblick}

Mit dem hier vorgestellten Fragebogen zu nationalen und sprachlichen Stereotypen haben wir bereits Untersuchungen in Deutschland, China, Russland und der Türkei durchgeführt. Dabei haben bisher über 900 Probanden in China, über 3500 in Russland und über 2000 in der Türkei die Fragen über Deutsche und Deutschland sowie die deutsche Sprache beantwortet. In Deutschland existieren bisher (Stand April 2015) über 3800 Antworten zu Russland und Russen, über $2500 \mathrm{zu}$ China und Chinesen und über $1000 \mathrm{zu}$ der Türkei und Türken. Es hat sich gezeigt, dass der von uns verwendete Fragebogen in allen vier Nationen nutzbar ist und die

\footnotetext{
${ }^{11}$ Den Mitgliedern der Forschungsgruppe SI.DE, die sich mit den chinesischen Aspekten unserer Erhebung beschäftigen, gilt unser Dank für die Übersetzungsarbeit und Übertragung. Dies sind Yi Li, Yue Qi und Ningjie Zhang.
} 
Begrifflichkeiten für alle benannten Ethnien verwendet werden können. Durch weitere Erhebungen sollen auch longitudinale Perspektiven eröffnet werden.

Erste vorläufige Auswertungen zeigen, dass es innerhalb der befragten ethischen Gruppen erhebliche Unterschiede in den Einstellungen gibt, wenn bei der Analyse der Gruppen die soziodemographischen Faktoren berücksichtigt werden. Dies ist bei den Antworten aus allen vier Kulturräumen zu beobachten. Besonders interessant ist auch die Betrachtung der in Deutschland lebenden Personen mit russischem und türkischem Migrationshintergrund im Vergleich zu den ,autochtonen“ Deutschen und im Vergleich zu den Russen und Türken in den Herkunftsländern.

Schon Sodhi und Bergius konnten zudem in ihrer Studie aufzeigen, dass es Unterschiede in den stereotypen Vorstellungen bei weiblichen und männlichen Probanden gab. Nach Voruntersuchungen mit deutschen und russischen Probanden mit jeweils über 500 Befragten haben wir festgestellt, dass die Faktoren „Alter“ und „Geschlecht“ - wie nicht anders zu erwarten war - auch hier zu Differenzierungen führen. In den seitdem von uns durchgeführten Befragungen werden weitere soziodemografischen Angaben erhoben, und zwar neben Alter und Geschlecht das Herkunftsland der Befragten und die Region, in der sie leben, der Bildungsabschluss und Kontakte zu dem jeweiligen „fremden“ Land. Da wir auch überprüfen wollen, welche Stereotype bei in Deutschland lebenden Personen mit Migrationshintergrund vorherrschen, fragen wir auch nach der Herkunft der Eltern. Bei Studierenden wird gefragt, welche Fachrichtung sie studieren. Wenn ausländische Studierende Deutsch als Fremdsprache oder Germanistik studieren, wird das ebenfalls registriert.

Am Ende der Bearbeitung der Eigenschaftenliste werden zusätzlich halboffene und offene Fragen zu den von den Probanden angekreuzten Merkmalen gestellt. Die Probanden werden gebeten, die fünf Eigenschaften zu benennen, die sie als am meisten zutreffend einschätzen und Beispiele zu geben, die diese Eigenschaften verdeutlichen.

Grundsätzlich haben wir uns entschlossen, unsere Untersuchungen online durchzuführen. Dies bietet aus unserer Sicht große Vorteile, da durch eine entsprechende Programmierung die Antwortoptionen nicht durchgehend zur Verfügung stehen und durch „Zurückblättern“ keine Veränderungen vorgenommen werden können, sondern die Probanden durch die Umfragen geführt werden. In Regionen oder bei Personen, die keinen Zugang zum Internet haben oder haben wollen, können wir jedoch auf die Paper-and-Pencil-Methode zurückgreifen und die vorgenommene Befragungen anschließend zur Auswertung in ein digitales System übertragen.

Sympathien und Antipathien gegenüber anderen Völkern verbinden sich häufig auch in Stereotypen und Einstellungen gegenüber der fremden Sprache. Deshalb ist auch von Interesse, von den Teilnehmern an unseren Befragungen ihre Einschätzungen zur deutschen Sprache zu erfahren. Dabei lehnen wir unsere Fragen an das Antonymen-Modell des Musikpädagogen Reinecke (1982) an. Wir erheben diese Sprach-Einstellungen mit einer Likert-Skala, die auf einer Skala von -5 bis +5 reicht.

Durch 22 gegensätzliche Adjektiv-Paare entsteht ein „Polaritätsprofil“ für das Deutsche (cf. Anhang). Neben sich leicht erschließenden Adjektiv-Paaren wie hart vs. weich, schön vs. hässlich und klar vs. verschwommen untersuchen wir momentan auch, ob andere metaphori- 
sche Ausdrücke wie z. B. müde vs. lebhaft, klagend vs. munter u. a. in den von uns untersuchten Kontaktsprachen ebenfalls benutzt werden können. Des Weiteren soll analysiert werden, ob bei Personengruppen, die eine eher negative Einstellung zu Deutschland und den Deutschen haben, sich diese negative Einstellung auch in der metaphorischen Bewertung der deutschen Sprache widerspiegelt.

\section{Literatur}

Apeltauer, Ernst (ed.) (2002): Interkulturelle Kommunikation. Deutschland - Skandinavien Großbritannien. Tübingen: Narr.

Baur, Rupprecht S./Ossenberg, Stefan/Zarudko, Marina (2013): „Deutsche und russische Stereotypen im Vergleich - Zur Erforschung von nationalen Bildern in unseren Köpfen“. In: Kellermeier-Rehbein, Birte/Haselhuber, Jakob/Schneider-Wiejowski, Karina (eds.): Vielfalt, Variation und Stellung der deutschen Sprache. Berlin/New York, de Gruyter: 549562.

Florack, Ruth (2007): Bekannte Fremde. Zu Herkunft und Funktion nationaler Stereotype in der Literatur. Tübingen: Niemeyer.

Forsa (2007): Das Russland-Bild der Deutschen. Wahrnehmung, Urteile und Stereotype. www.wingas.de/fileadmin/Presse_PDF/2007/Charts_PK_Druckversion.pdf [20.02.2014].

Gilbert, Gustave M. (1951): "Stereotype persistence and change among college students". The Journal of Abnormal and Social Psychology 46/2: 245-254.

Goethe-Institut (2011): „Die Deutschland-Liste. Was denkt Europa über Deutschland?“ www.goethe.de/ins/be/prj/dli/deindex.htm [10.12.2015].

Grünewald, Matthias (2005): Bilder im Kopf. Eine Longitudinalstudie über die Deutschlandund Deutschenbilder japanischer Deutschlernender. München: Iudicium.

Hahn, Hans Henning/Mannová, Elena (eds.) (2007): Nationale Wahrnehmungen und ihre Stereotypisierung. Beiträge zur historischen Stereotypenforschung. Frankfurt a. M.: Lang.

Karlins, Marvin/Coffman, Thomas L./Walters, Gary (1969): “On the fading of social stereotypes: Studies in three generations of college students". Journal of Personality and Social Psychology 13/1: 1-16.

Katz, Daniel/ Braly, Kenneth (1933): "Stereotypes of one Hundred College Students". Journal of Abnormal and Social Psychology 28: 280-290.

Keller, Gottfried (1986): „Das Deutschlandbild amerikanischer, britischer und französischer Schüler im kulturkundlichen Unterricht". Neusprachliche Mitteilungen aus Wissenschaft und Praxis 39/4: 209-217.

Kucharczyk, Jacek et al. (2013): Im Osten was Neues? Das Bild Polens und Russlands in Deutschland 2013. www.bertelsmann-stiftung.de/fileadmin/files/BSt/Presse/imported/ downloads/xcms_bst_dms_38167_38168_2.pdf [10.12.2015].

Lippmann, Walter (1989): Die öffentliche Meinung. Übersetzung von „Public Opinion“ (1922). Bochum: Brockmeyer.

Löschmann, Martin/Stroinska, Magda (1998): „Stereotype, Stereotype und kein Ende“. In: Löschmann, Martin (ed.): Stereotype im Fremdsprachenunterricht. Frankfurt a. M., Lang: 7-33. 
Rheingold Salon (2012): Das Doppelleben der Deutschen. Die OeTTINGER DeutschlandStudie. Www.rheingold-salon.de/grafik/veroeffentlichungen/Deutschland_Studie_reader_ 2012-09-19.pdf [10.12.2015].

Reinecke, Hans-Peter (1982): „Kommunikative Musikpsychologie. Grundlagen der Musiktherapie und Musikpsychologie“. In: Harrer, Gerhart (ed.): Grundlagen der Musiktherapie und Musikpsychologie. Stuttgart, Fischer: 111-122.

Sodhi, Kripal Singh/Bergius, Rudolf (1953): Nationale Vorurteile. Berlin: Duncker und Humblot.

Springer, Philipp/Jahn, Peter (2008): Unsere Russen - unsere Deutschen. Bilder vom Anderen 1800 bis 2000. Berlin: Links. 
Anhang A: Eigenschaftenliste (Baur/Ossenberg 2014: https://side.uni-due.de/merkmalliste [06.04.2016]).

\begin{tabular}{|c|c|c|c|c|c|}
\hline 001. & sprachbegabt & 048. & natürlich & 095. & unberechenbar \\
\hline 002. & gute Politiker & 049. & nationalstolz & 096. & gute Wissenschaftler \\
\hline 003. & zuverlässig & 050. & konservativ & 097. & militaristisch \\
\hline 004. & ruhmsüchtig & 051. & naiv & 098. & oberflächlich \\
\hline 005. & anständig & 052. & diplomatisch & 099. & bequem \\
\hline 006. & schlechte Demokraten & 053. & schlechte Politiker & 100. & gute Hausfrauen \\
\hline 007. & großzügig & 054. & Massenmenschen & 101. & brutal \\
\hline 008. & gut gewachsen & 055. & anspruchsvoll & 102. & sentimental \\
\hline 009- & pflichtbewusst & 056. & gute Organisatoren & 103. & diszipliniert \\
\hline 010. & tolerant & 057. & rekordsüchtig & 104. & Handelsvolk \\
\hline 011. & materiell eingestellt & 058. & schönheitsliebend & 105. & weltmännisch \\
\hline 012. & konventionell & 059. & bestechlich & 106. & unsoldatisch \\
\hline 013. & schmutzig & 060. & ausdauernd & 107. & rachsüchtig \\
\hline 014. & handwerklich begabt & 061. & autoritätshörig & 108. & korrekt \\
\hline 015- & kultiviert & 062. & gute Demokraten & 109. & Klassenunterschiede \\
\hline 016. & heimatliebend & 063. & zielstrebig & 110. & revolutionär \\
\hline 017. & Herrenvolk & 064. & Idealisten & 111. & willensstark \\
\hline 018. & reserviert & 065. & gute Ärzte & 112. & dekadent \\
\hline 019. & tapfer & 066. & gute Techniker & 113. & fernsehbegeistert \\
\hline 020. & höflich & 067. & philosophische Lebenshaltung & 114. & traditionsgebunden \\
\hline 021. & Volk von Bauern & 068- & rassebewusst & 115. & stolz \\
\hline 022. & großspurig & 069. & arm & 116. & fleißig \\
\hline 023. & jähzornig & 070. & ungebildet & 117. & fortschrittlich \\
\hline 024. & stur & 071. & nüchtern & 118. & humorvoll \\
\hline 025. & misstrauisch & 072. & anspruchslos & 119. & hübsche Frauen \\
\hline 026. & tierliebend & 073. & fanatisch & 120. & musikalisch \\
\hline 027. & gute Soldaten & 074. & individualistisch & 121. & dankbar \\
\hline 028. & Zusammengehörigkeitsgefühl & 075. & reich & 122. & trinkfreudig \\
\hline 029. & sportlich & 076. & impulsiv & 123. & friedliebend \\
\hline 030. & unzuverlässig & 077. & sparsam & 124. & emotional \\
\hline 031. & religiös intolerant & 078. & religiös & 125. & rational \\
\hline 032. & Überschätzung des Fremden & 079. & abergläubisch & 126. & gesellig \\
\hline 033. & leichtlebig & 080. & sauber & 127. & arbeitsfreudig \\
\hline 034. & Dichter und Denker & 081. & arrogant & 128. & kriminell \\
\hline 035. & künstlerisch & 082. & gastfreundlich & 129. & pünktlich \\
\hline 036. & kinderlieb & 083. & modisch & 130. & umweltbewusst \\
\hline 037. & unterwürfig & 084. & rücksichtslos & 131. & ängstlich \\
\hline 038. & primitiv & 085. & streitsüchtig & 132. & freundlich \\
\hline 039. & neidisch & 086. & umgänglich & 133. & distanziert \\
\hline 040. & familienorientiert & 087. & gründlich & 134. & großherzig \\
\hline 041. & bürokratisch & 088. & verschlossen & 135. & ehrlich \\
\hline 042. & maßlos & 089. & kameradschaftlich & 136. & friedlich \\
\hline 043. & eitel & 090. & treu & 137. & attraktive Männer \\
\hline 044. & Volk der Zukunft & 091. & freiheitsliebend & 138 & ordentlich \\
\hline 045. & intelligent & 092. & selbstbewusst & 139. & fremdenfeindlich \\
\hline 046. & faul & 093. & lässig & 140. & rassistisch \\
\hline 047. & geizig & 094. & hinterlistig & & \\
\hline
\end{tabular}


Anhang B: Polaritätsprofil (Baur/Ossenberg 2014: https://side.uni-due.de/polaritaet [06.04.2016]).

\begin{tabular}{|c|c|c|c|c|c|c|c|c|c|c|c|c|}
\hline & -5 & -4 & -3 & -2 & -1 & 0 & +1 & +2 & +3 & +4 & +5 & \\
\hline hässlich & & & & & & & & & & & & schön \\
\hline unangenehm & & & & & & & & & & & & angenehm \\
\hline aggressiv & & & & & & & & & & & & friedlich \\
\hline fremd & & & & & & & & & & & & vertraut \\
\hline rauh & & & & & & & & & & & & glatt \\
\hline hart & & & & & & & & & & & & weich \\
\hline unregelmäßig & & & & & & & & & & & & regelmäßig \\
\hline klagend & & & & & & & & & & & & munter \\
\hline kühl & & & & & & & & & & & & gefühlvoll \\
\hline verschwommen & & & & & & & & & & & & klar \\
\hline blass & & & & & & & & & & & & farbig \\
\hline passiv & & & & & & & & & & & & aktiv \\
\hline aufdringlich & & & & & & & & & & & & zurückhaltend \\
\hline dumpf & & & & & & & & & & & & klingend \\
\hline kalt & & & & & & & & & & & & warm \\
\hline traurig & & & & & & & & & & & & froh \\
\hline müde & & & & & & & & & & & & lebhaft \\
\hline langsam & & & & & & & & & & & & schnell \\
\hline dunkel & & & & & & & & & & & & hell \\
\hline robust & & & & & & & & & & & & zart \\
\hline unmelodisch & & & & & & & & & & & & melodisch \\
\hline \multirow[t]{2}{*}{ träge } & & & & & & & & & & & & dynamisch \\
\hline & -5 & -4 & -3 & -2 & -1 & 0 & +1 & +2 & +3 & +4 & +5 & \\
\hline
\end{tabular}

